\title{
Bitopic ligands: all-in-one orthosteric and allosteric
} Maud Kamal $^{1,2}$ and Ralf Jockers ${ }^{1,2 *}$

\author{
Addresses: ${ }^{1}$ Institut Cochin, Université Paris Descartes, CNRS (UMR 8104), Paris, France; ${ }^{2}$ Inserm U567, Paris, France \\ * Corresponding author: Ralf Jockers (ralf.jockers@inserm.fr) \\ FI000 Biology Reports 2009, I:77 (doi:I0.34I0/BI-77) \\ The electronic version of this article is the complete one and can be found at: http://FI000.com/Reports/Biology/content/I/77
}

\begin{abstract}
Natural ligands of G-protein-coupled receptors interact with the orthosteric ligand binding site, as do most of the classical synthetic ligands. The discovery of ligands targeting different, allosteric binding sites considerably expanded the repertoire of G-protein-coupled receptor ligands. More recently, bitopic ligands have been described that target both orthosteric and allosteric sites at the same time.
\end{abstract}

\section{Introduction and context}

G-protein-coupled receptors (GPCRs) constitute the largest family of membrane proteins and are known to respond to a plethora of different stimuli by activating numerous intracellular signaling pathways. Because of their involvement in a variety of biological processes, GPCRs are the target of more than $50 \%$ of currently marketed drugs [1]. To date, most of the drugs targeting GPCRs are known to interact with the orthosteric site that is, the endogenous ligand-binding pocket [2]. These orthosteric ligands need, however, to overcome many limitations, such as decreased selectivity, insufficient clinical efficacy, and undesirable effects on receptor regulation [3].

The past decade witnessed the discovery of new pharmaceutical compounds that modulate receptor function by targeting allosteric sites. Allosteric sites are, by definition, domains topographically distinct from the orthosteric binding pocket [4]. These allosteric modulators are very promising pharmaceutical drugs devoid of many of the disadvantages of orthosteric ligands. In fact, these allosteric compounds show higher subtype selectivity (allosteric sites are generally less conserved than orthosteric sites), often have a 'safer' pharmacological profile (exert their effects only in the presence of endogenous ligands) and, in some cases, do not induce receptor desensitization [4].
There are three general categories of allosteric modulators [3] (Figure 1a): first, modulators affecting the binding affinity of orthosteric ligands, such as AMNO82, which is responsible for the incomplete inhibition of orthosteric antagonist binding to the metabotropic glutamate (mGlu) 7 receptor, mGlu7R [5]; and second, modulators affecting orthosteric ligand efficacy. One example is CGP7930, which is known to enhance gamma-aminobutyric acid receptor $\mathrm{B}\left(\mathrm{GABA}_{\mathrm{B}}\right)$-mediated GTP $\gamma \mathrm{S}$ (guanosine 5'-O-[gamma-thio]triphosphate) binding [6]. Third, allosteric agonists and inverse agonists, known to engender a unique GPCR conformation that alters receptor signaling in the absence of orthosteric ligands $[3,4]$. For example, MPEP (2-methyl6-(phenylethynyl)pyridine) acts as a negative allosteric modulator of mGlu5R by inhibiting inositol phosphate production in the absence of an agonist [7]. Another example is AC42, which was first identified as a selective agonist of the M1 muscarinic acetylcholine receptor (M1AChR) [8]; however, mutation studies have implied that this ligand activates the receptor via an allosteric site [9].

\section{Recent advances}

Recently, a new class of ligands, termed bitopic or dualsteric ligands, which simultaneously target orthosteric and allosteric sites, emerged (Figure 1b). The development of bitopic ligands is based on the idea of combining 
Figure I. Different binding modes of orthosteric, allosteric, and bitopic ligands

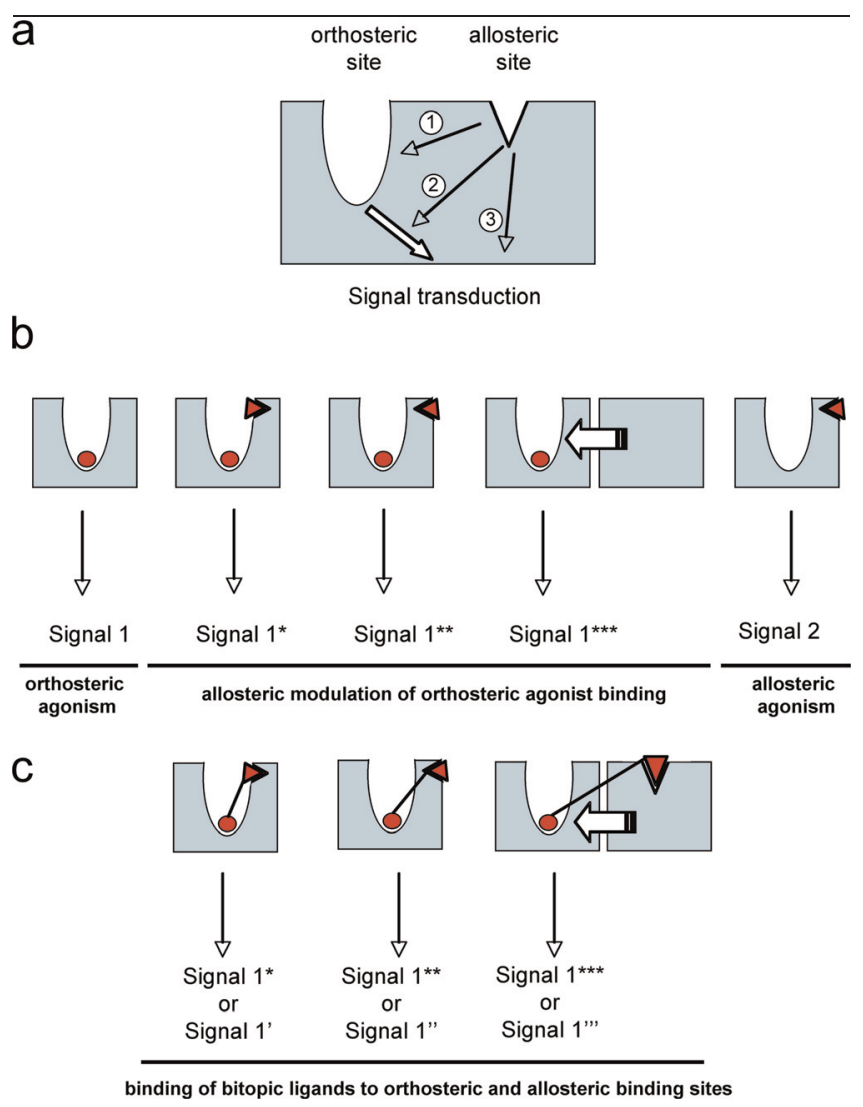

(a) An allosteric ligand may modulate binding ( 1 ) and signaling (2) of orthosteric ligands or (3) induce signal transduction by its own (allosteric agonists) (adapted from Langmead and Christopoulos [3]). $(b, c)$ Comparison of different binding modes of orthosteric (red circle) and allosteric (red triangle) ligands as monovalent ligands (b) or bivalent bitopic ligands (c). Binding of orthosteric ligands induces Signal I, which can be modulated by allosteric ligands binding to sites that are close to or distant from the orthosteric binding site, generating Signal I* or Signal I**.

Alternatively, the signal generated by the orthosteric ligand in one protomer can be allosterically modulated by the other protomer within a GPCR dimer, generating Signal I***. Allosteric agonists can induce signaling by their own in the absence of orthosteric ligands (Signal 2). Signals I*, I**, and I**** can also be generated by bitopic ligands. In addition, bitopic ligands could induce signals (Signals I', I', and I'”) that are specific for these ligands and not observed upon simultaneous stimulation with monovalent orthosteric and allosteric ligands.

high affinity (via orthosteric sites) with high selectivity (via allosteric sites). Until now, muscarinic acetylcholine receptors (mAChRs) have proved to be a particularly fruitful receptor model for the development and characterization of bitopic ligands. In 2004, Tahtaoui et al. [10] synthesized seven fluorescent derivatives of the BODIPY-labeled pirenzipine, an M1AChR antagonist, and used fluorescent resonance energy transfer technology to study receptor-ligand interactions. The authors showed that these analogs might interact with both the acetylcholine (orthosteric) and brucine (allosteric) binding domains of the M1AChR, therefore behaving as potential bitopic ligands.

The literature also reports the design of hybrid molecules from allosteric modulators of W84-type compounds and orthosteric antagonists or agonists of mAChRs to obtain subtype selective muscarinic ligands with agonistic or antagonistic properties [11].

In a recent publication [12], Christopoulos and colleagues elegantly showed that McN-A-343, a selective M2AChR partial agonist, is actually a bitopic ligand. Using several different binding and functional assays, the authors showed that McN-A-343 is composed of an orthosteric agonist coupled to an allosteric modulator (3-chlorophenylcarbamate moiety). Truncated derivatives of McN-A-343 retaining the chlorophenylcarbamate moiety were positive modulators of the orthosteric antagonist $\mathrm{N}$-[3H]methylscopolamine in radioligand binding assays, but in functional assays, such as M2AChR-mediated ERK1/2 (extracellular signal-regulated kinase 1/2) phosphorylation, they acted as negative modulators of agonist efficacy.

Finally, in 2009, Antony et al. [13] presented a novel approach to pharmacologically design subtype and signaling selective receptor agonists, once again using the mAChRs as templates. Their paper reports the synthesis of two hybrids fusing a highly potent oxotremorine-like orthosteric activator with M2-selective bis(ammonio)alkane-type allosteric fragments. Radioligand binding in wild-type and mutant receptors supplemented by receptor docking simulations showed an M2-selective and true allosteric/orthosteric binding. Moreover, G-protein activation, in this context, was mediated by the orthosteric moieties. Interestingly, hybrid compounds showed ligand-biased signaling properties.

\section{Future directions}

Due to the unique features of bitopic ligands, their number is likely to expand in the near future. This may include the re-examination of already existing ligands, as was the case with McN-A-343 [12], and the de novo design of bitopic ligands as shown by Antony et al. [13]. Where can we expect to see the fastest progress? The number of already existing ligands that might turn out to be bitopic is difficult to estimate and will strongly depend on the degree of information available for these putative bitopic molecules. However, most progress is expected to come from newly designed ligands. 
The design of new bitopic ligands requires extensive knowledge about orthosteric and allosteric ligands available for a given GPCR as well as its ligand binding sites. This may explain the fact that bitopic ligands have predominantly been described for muscarinic receptors, for which detailed knowledge on allosteric binding sites and ligands is indeed available. Once allosteric ligands have been identified, the choice of an optimal linker has to be considered. The extensive literature on the design and synthesis of bivalent ligands intended to target two orthosteric ligand binding sites within a GPCR dimer will be a rich source of inspiration for the synthesis of bitopic ligands [14]. As illustrated by compound McN-A343 and hybrid compounds described by Antony et al. [13], linker length can vary considerably even for the same receptor. Obviously, the better the structural knowledge about orthosteric and allosteric binding sites, the easier the rational design of the linker (chemical nature and length of linker, linker attachment point to pharmacophores) will be.

Recently solved GPCR structures will assist the design and synthesis of bitopic ligands that bind to identified orthosteric and allosteric binding pockets. Such GPCR structures have not only provided detailed knowledge about orthosteric binding pockets, but also revealed the important role of extracellular receptor domains in guiding small molecular weight ligands to their high affinity ligand binding pockets within the transmembrane domain [15]. These 'ligand entrance channels' are interesting new targets for allosteric modulation by bitopic ligands. Finally, the concept of bitopic ligands may also be extended to GPCR dimers, where the orthosteric and allosteric pharmacophores bind to two different protomers in the receptor dimer (Figure 1b) [14].

\section{Abbreviations}

BODIPY, boron-dipyrromethene; ERK1/2, extracellular signal-regulated kinase $1 / 2 ; \mathrm{GABA}_{\mathrm{B}}$, gamma-aminobutyric acid receptor B; GPCR, G-protein-coupled receptor; GTP $\gamma$, guanosine 5'-O-[gamma-thio]triphosphate; M1AChR, M1 muscarinic acetylcholine receptor; mAChR, muscarinic acetylcholine receptor; mGluR, metabotropic glutamate receptor; MPEP, 2-methyl-6(phenylethynyl)pyridine.

\section{Competing interests}

The authors declare that they have no competing interests.

\section{Acknowledgements}

This work was supported by grants from the Fondation Recherche Médicale ('Equipe FRM'), SERVIER, and the Institut National de la Santé et de la Recherche Médicale (INSERM).

\section{References}

I. Kenakin T: G-protein coupled receptors as allosteric machines. Receptors Channels 2004, 10:51-60.

2. Neubig RR, Spedding M, Kenakin T, Christopoulos A: International Union of Pharmacology Committee on Receptor Nomenclature and Drug Classification. XXXVIII. Update on terms and symbols in quantitative pharmacology. Pharmacol Rev 2003, 55:597-606.

3. Langmead CJ, Christopoulos A: Allosteric agonists of 7TM receptors: expanding the pharmacological toolbox. Trends Pharmacol Sci 2006, 27:475-8I.

4. May LT, Leach K, Sexton PM, Christopoulos A: Allosteric modulation of $\mathbf{G}$ protein-coupled receptors. Annu Rev Pharmacol Toxicol 2007, 47:I-5I.

5. Mitsukawa K, Yamamoto R, Ofner S, Nozulak J, Pescott O, Lukic S, Stoehr N, Mombereau C, Kuhn R, McAllister KH, van der Putten H, Cryan JF, Flor PJ: A selective metabotropic glutamate receptor 7 agonist: activation of receptor signaling via an allosteric site modulates stress parameters in vivo. Proc Natl Acad Sci U S A 2005, 102: 187|2-7.

6. Urwyler S, Mosbacher J, Lingenhoehl K, Heid J, Hofstetter K, Froestl W, Bettler B, Kaupmann K: Positive allosteric modulation of native and recombinant gamma-aminobutyric acid(B) receptors by 2,6-di-tert-butyl-4-(3-hydroxy-2,2-dimethyl-propyl)-phenol (CGP7930) and its aldehyde analog CGPI350I. Mol Pharmacol 2001, 60:963-71.

7. Porter RH, Jaeschke G, Spooren W, Ballard TM, Buttelmann B, Kolczewski S, Peters JU, Prinssen E, Wichmann J, Vieira E, Muhlemann A, Gatti S, Mutel V, Malherbe P: Fenobam: a clinically validated nonbenzodiazepine anxiolytic is a potent, selective, and noncompetitive $\mathrm{mGlu} 5$ receptor antagonist with inverse agonist activity. J Pharmacol Exp Ther 2005, 3 I 5:7 I I-2I.

8. Spalding TA, Trotter C, Skjaerbaek N, Messier TL, Currier EA, Burstein ES, Li D, Hacksell U, Brann MR: Discovery of an ectopic activation site on the $\mathrm{M}(\mathrm{I})$ muscarinic receptor. Mol Pharmacol 2002, 6 I: 1297-302.

9. Langmead CJ, Fry VA, Forbes IT, Branch CL, Christopoulos A, Wood MD, Herdon H]: Probing the molecular mechanism of interaction between 4-n-butyl-I-[4-(2-methylphenyl)-4-oxoI-butyl]-piperidine (AC-42) and the muscarinic $M(I)$ receptor: direct pharmacological evidence that $\mathrm{AC}-42$ is an allosteric agonist. Mol Pharmacol 2006, 69:236-46.

I0. Tahtaoui C, Parrot I, Klotz P, Guillier F, Galzi JL, Hibert M, Ilien B: Fluorescent pirenzepine derivatives as potential bitopic ligands of the human MI muscarinic receptor. J Med Chem 2004, 47:4300-I5.

II. Holzgrabe U, De Amici M, Mohr K: Allosteric modulators and selective agonists of muscarinic receptors. J Mol Neurosci 2006, 30:165-8.

12. Valant C, Gregory KJ, Hall NE, Scammells PJ, Lew MJ, Sexton PM, Christopoulos A: A novel mechanism of $\mathbf{G}$ protein-coupled receptor functional selectivity. Muscarinic partial agonist McN-A-343 as a bitopic orthosteric/allosteric ligand. J Biol Chem 2008, 283:29312-2I.

13. Antony J, Kellershohn K, Mohr-Andra M, Kebig A, Prilla S, Muth M, Heller E, Disingrini T, Dallanoce C, Bertoni S, Schrobang J, Trankle C, Kostenis E, Christopoulos A, Holtje HD, Barocelli E, De Amici M, Holzgrabe U, Mohr K: Dualsteric GPCR targeting: a novel route to binding and signaling pathway selectivity. Faseb J 2009, 23:442-50.

FI000 Factor 6.0 Must Read Evaluated by Ralf Jockers 31 Jul 2009

14. Berque-Bestel I, Lezoualc'h F, Jockers R: Bivalent ligands as specific pharmacological tools for $\mathbf{G}$ protein-coupled receptor dimers. Curr Drug Discov Technol 2008, 5:312-8.

15. Kobilka B, Schertler GF: New G-protein-coupled receptor crystal structures: insights and limitations. Trends Pharmacol Sci 2008, 29:79-83. 